\title{
Grupo de Trabajo de la OMS sobre "evaluación en Salud Mental": Reflexiones tras una experiencia de tres años
}

\footnotetext{
* Profesor de Psiquiatría, Hospitales Universitarios de Ginebra

SUIZA

** Profesor de Psiquiatría, Director del Grupo de Trabajo, Centro Colaborador para la Salud y los factores psicosociales y psicobiológicos, Bruselas BÉLGICA

*** Profesor de Psiquiatría. Universidad de Leipzig

ALEMANIA

**** Departamento de Psiquiatría, Hospital General Ruzinov, Bratislava SLOVAKIA

***** Instituto de Psiquiatría y Neurología, Varsovia POLONIA

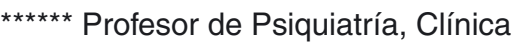
Psiquiátrica, Facultad de Medicina Universidad de Lübeck ALEMANIA
}

\author{
José Guimón, MD* \\ Franz. C.J. Baro, MD**

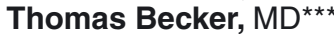 \\ Peter Breier, MD**** \\ Jan Czeslaw Czabala, $\mathrm{PhD}^{\star \star \star \star \star}$

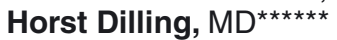 \\ John H. Henderson, MD******* \\ Leen Meulenberg, MA $\mathrm{A}^{\star \star \star \star \star \star \star \star *}$ \\ Bogdana Tudorache, $\mathrm{PhD} \mathrm{D}^{\star \star \star \star \star \star \star \star \star \star}$ \\ Vassilly S. YastreboV ${ }^{\star \star \star \star \star \star \star \star \star \star * \star}$ \\ Wolfgang Rutz, MD $\mathrm{D}^{\star \star \star \star \star \star \star \star \star \star \star \star \star ~}$ \\ Anke Bramesfeld, MD ${ }^{\star \star \star \star \star \star \star \star \star \star \star \star \star \star ~}$
}

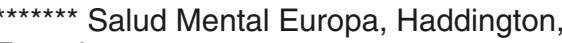
Escocia

REINO UNIDO

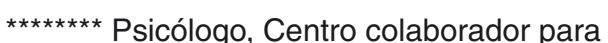
la Salud y los factores psicosociales y psicobiológicos, Bruselas BÉLGICA

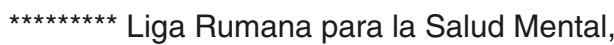

Bucarest

RUMANIA

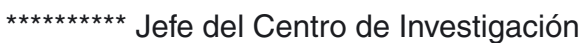
de los Sistemas de Soporte en Salud Mental, Centro Científico para la Salud Mental, Moscú

FEDERACIÓN RUSA

********** Consejero Regional de Salud Mental, Oficina Regional de la OMS para Europa, Copenhague

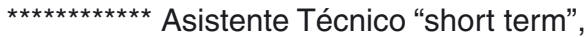
Oficina Regional de la OMS para Europa Copenhague

DINAMARCA 
RESUMEN - La Oficina Regional para Europa de la Organización Mundial de la Salud (OMS) en Copenague estableció un grupo de trabajo sobre evaluación en Salud Mental formado por expertos en Psiquiatría, los cuales, junto con los centros colaboradores de la OMS, prestan asistencia a una red Europea de Salud Mental constituido por 47 representantes nacionales para la Salud Mental nombrados por los diferentes ministerios. Este artículo discute las evaluaciones que se han hecho en siete estados de la Europa del Este y del Sureste durante los tres años de vida del grupo de trabajo. La mayoría de los instrumentos de evaluación que se utilizan en la actualidad exigen estudios de campo largos y costosos, que rara vez son posibles en el contexto de este tipo de evaluación. En el futuro se deberá hacer un esfuerzo considerable para consensuar procedimientos simples y fiables para evaluar estos parámetros y controlar la evolución. Por lo general, las evaluaciones descritas aumentaron el interés y la sensibilidad por las cuestiones de Salud mental, colocando de esta manera estas cuestiones en el centro de la política en la mayoría de los países concernidos.

Este artículo es un resumen de la experiencia adquirida por el "Grupo de Trabajo Europeo sobre evaluación de la salud mental" de la OMS durante los tres últimos años, de sus objetivos, de su metodología y del efecto que han tenido los diversos proyectos ya empezados, a la vez en los paises respectivos y en el propio grupo de trabajo.

\section{El Grupo de Trabajo para la Evaluación de la Salud Mental}

\section{La creación}

El programa de Salud Mental de la Oficina Regional Europea de la OMS identificó en 1998 la necesidad de aconsejar acerca de la puesta en marcha de nuevas políticas en Salud Mental, consejo solicitado con frecuencia por diferentes estados (OMS 1999), principalmente por los paises de Europa del Este, que en la actualidad están sintiendo la necesidad urgente de modificar sus servicios de Salud Mental (WHO 1999). Se creó un grupo de trabajo para evaluaciones de la Salud Mental que incluía a los miembros autores de este artículo. Se decidió que, para realizar sus objetivos, el grupo de trabajo debería estar en contacto con los cuarteles generales de la OMS en Ginebra, las otras redes de la Comisión Regional, otras organizaciones en la Unión Europea, la Federación Mundial de la Salud Mental (WFMH) y la Asociación Mundial de Psiquiatría.

El grupo de trabajo se reune oficialmente dos veces al año, acogido siempre por uno de los miembros. De esta forma, cada miembro y su gobierno tiene la oportunidad de utilizar la presencia de este grupo de expertos internacionales para su propio país mediante conferencias de prensa, reuniones ministeriales, consultas, etc.

\section{El Modelo de Salud Mental}

La OMS defiende un modelo preciso de promoción y de asistencia en salud mental (Hardmann y Joughin 1998, Mattson Marlin 1992):

1. La asistencia en Salud Mental ha de hacerse dentro de un contexto de participa- 
ción, implicando a los clientes y a sus familias en la toma de decisiones.

2. El tamaño de los hospitales psiquiátricos debería reducirse y minimizarse el tratamiento de los pacientes hospitalizados

3. Cuando ello sea posible, el tratamiento hospitalario debe hacerse en el hospital general.

4. La asistencia hospitalaria y ambulatoria debería proporcionarse lo más cerca posible de la población a la que va dirigida, dentro de servicios de Salud Mental basados en la comunidad.

5. Los servicios ambulatorios y los centros de día deberían estar ampliamente disponibles e incluirse en la asistencia sanitaria primaria.

6. Se debería desarrollar una asistencia extra muros, no médica, realizada por asistentes sociales, enfermeras, etc.

7. Debería seguirse el principio del trabajo en equipo multidisciplinario.

8. La Salud Mental concierne a todos los sectores de la Sociedad y todos ellos son responsables de ella.

\section{Los objetivos del grupo de trabajo para la evaluación de la Salud Mental}

De acuerdo con esta política de la OMS, el grupo de trabajo se planteó los siguientes objetivos:

1. Recoger datos sobre la Salud mental basados en las pruebas ("evidence based").

2. Publicar un artículo de situación sobre el estado de la cuestión en Europa.

3. Coordinar la investigación que ha de llevarse a cabo.
4. Desarrollar estrategias basadas en las pruebas; llevarlas a cabo en uno o dos lugares como "buenos ejemplos".

5. Preparar y tener una reunión sobre el tema en el año 2002.

6. Desarrollar un plan de acción sobre el tema a presentar y a adoptar en esa reunión.

7. Integrar el trabajo hecho por el grupo de trabajo en la preparación de la conferencia ministerial de la OMS sobre salud mental propuesta para 2003-4.

\section{Las actividades}

\section{Las demandas y los métodos}

El grupo de trabajo ha respondido a demandas de evaluación mediante visitas a los países y la elaboración de informes y de recomendaciones. Sin embargo, antes de adquirir cualquier compromiso, tuvo que clarificar cuáles eran las instancias que pedían una evaluación y cual sería el alcance y los límites de la intervención que podía ofrecerse. En realidad, una evaluación podía ser solicitada por organizaciones supranacionales (tales como las Naciones Unidas o la propia OMS), o por algunas instancias privadas (tales como la Asociación Mundial de Psiquiatría, y organizaciones no gubernamentales), pero hasta ahora, las consultas han sido requeridas (directa o indirectamente) por los Estados miembros, que desean cambiar su política en Salud Mental y confían en la OMS porque tiene el conocimiento y la objetividad requeridos. Obviamente, el tipo de evaluación que ha de llevarse a cabo depende del origen y de la razón de la demanda.

En el cumplimiento de su misión, el grupo de trabajo recoge, recurriendo a las fuentes de datos locales e internacionales y durante las visitas in situ, la información siguiente: 
1. Un panorama de la situación de los servicios de salud mental en el país.

2. La evaluación de las necesidades de la población de pacientes.

3. Los planes de promoción de la Salud Mental existentes.

4. Las inversiones que el país realiza en Salud Mental.

5. El grado de interrelación entre los componentes físicos y mentales de la Asistencia médica y social.

6. Evaluación de los servicios de Salud Mental existentes.

7. Evaluación de la calidad de la gestión.

8. Evaluación de la legislación sobre Derechos Humanos y equidad.

Sin embargo, el alcance y la metodología de las intervenciones dependen en gran parte de la definición precisa de la demanda (Freeman et al. 2000). Así las evaluaciones pueden llevarse a cabo mediante visitas de evaluación y procedimientos de acreditación; pueden centrarse en una población diana determinada o en una toda una organización de salud; pueden utilizar instrumentos de evaluación de necesidades existentes desde hace tiempo o de reciente creación, manuales operativos e indicadores de estigma, cuestiones de discriminación y de equidad, etc.

Hasta ahora, el grupo de trabajo ha hecho evaluaciones en Polonia, Kyrgyzstan, Rumania, Lituania y Eslovenia y visitas cortas a Rusia y Georgia con la finalidad de reestablecer los contactos e introducir programas de Salud Mental en el contexto de las políticas re-emergentes, explorar la situación actual en Salud Mental y fomentar el interés por encontrar fondos para los diferentes proyectos. Los límites de tiempo y de presupuesto son proporcionales a la tarea, y la estrategia varía desde la simple consulta de datos aportados por el cliente hasta costosos estudios epidemiológicos en profundidad. En Rumania, por ejemplo, se hizo una evaluación bastante completa que permitió la obtención de datos sobre todos los campos relativos a la salud mental. En otras evaluaciones, uno o varios miembros (en el caso de Rusia todo el grupo de trabajo) visitaron los países, en misiones de "hallazgo de hechos" explorando (WHO 1998) algunas áreas específicas. Se reunieron con políticos, profesionales de la Salud Mental y representantes de organizaciones activas en el área. Además visitaron servicios de Salud Mental, principalmente hospitales e instituciones educativas y de investigación. Por supuesto, el origen de los fondos es una cuestión crítica en términos de imparcialidad y de eficacia de la tarea. Los resultados de estas evaluaciones se publicarán en otro lugar en un artículo separado y con más detalle. Un resultado positivo de todas estas actividades ha sido el poner las cuestiones de salud mental en el corazón de la política. Por lo general se ha logrado un aumento del interés y de la sensibilidad hacia las cuestiones de Salud Mental lo que ha llevado algunos países a solicitar evaluaciones más profundas. Otros países, como los siete que componen el "Pacto de estabilidad" del Sureste Europeo (los países llamados balcánicos) han solicitado una evaluación y miembros del grupo de trabajo han trabajado ya con representantes de todos los países para elaborar un cuestionario común de evaluación.

\section{Redes}

En los tres últimos años ha habido un intercambio continuo y productivo en temas de planificación, con la Federación Mundial por la Salud Mental (WFMH) "Mental Health Europe" y la Asociación Mundial de Psiquiatría (WPA). También ha existido una cooperación bastante intensa con la oficina 
regional de la Salud en Copenague, por ejemplo, en la revisión de datos de la prestación de servicios de salud mental en Europa, recogidos en 1994. Se contacta con frecuencia a los miembros de los grupos de trabajo y a las redes de la Oficina Regional y se ha formado un grupo compuesto por consejeros y expertos temporales en diferentes áreas de la Salud Mental.

El grupo de trabajo se ha implicado activamente en actividades de "concienciación", en especial durante el proyecto "2001, año de la Salud Mental" patrocinado por la OMS y las Naciones Unidas y ha invitado a todos los ministerios de la Salud europeos a un congreso pan-Europeo sobre Salud Mental, que tuvo lugar en octubre 2001 y fue acogido por el gobierno de Bélgica. En una reciente reunión OMS/Unión Europea, que también tuvo lugar en Bruselas, sobre la necesidad de la evaluación del impacto de la Salud Mental, se decidió extender el enfoque de la evaluación del grupo de trabajo a otros países europeos. También se han concertado acuerdos de cooperación con la "Geneva Initiative on Psychiatry" (GIP) para promover reformas en salud mental en países de Europa del Este.

\section{Interrelación entre los centros colaboradores de la OMS en Europa}

Se han organizado dos reuniones de los "centros colaboradores para la Salud Mental" de la OMS, en Copenague en 1999 y en Lille en 2001 y han tenido lugar más de seis reuniones de este grupo de trabajo, mejorando la cohesión grupal. En 1999, se pidió a los 51 Estados Miembros de la UE que nombraran a un representante nacional para el programa de salud mental de la OMS en la Comisión Regional. Hasta ahora, 47 países lo han designado. Los miembros de estas redes y de estos grupos de trabajo constituyen actualmente una importante red Europea en Salud Mental de la OMS.

\section{Compilación de Datos Nacionales}

En septiembre 1998, la Oficina Regional de la OMS adoptó un nuevo sistema de información sanitaria que incluía datos acerca de la mortalidad, la incidencia de enfermedades contagiosas, las organizaciones internacionales, las agencias y las publicaciones y las estadísticas sanitarias que se han compilado en tres bases de datos. Se halló que los datos fiables sobre Salud Mental son insuficientes. La labor de este grupo de trabajo está aportando en este campo valiosa información cuantitativa pero principalmente cualitativa.

\section{Amplitud y limitaciones}

A los tres años del comienzo de las evaluaciones y a la vista de los resultados mencionados con anterioridad, podemos hacer algunos comentarios sobre el alcance y las lagunas de este tipo de empresa:

\section{Evaluación de las necesidades del paciente individual}

La tendencia en la oferta de servicios de Salud Mental es actualmente la de hacer programas, "dirigidos hacia las necesidades" en lugar de "dirigidos hacia los servicios". Sin embargo, en el proceso de nuestras evaluaciones, nos faltaba el consenso en cuanto a los instrumentos para identificar los diferentes tipos de necesidades de una población determinada. En efecto, los instrumentos existen- 
tes (WHO 1996) se centran en los individuos de determinadas "poblaciones diana" y la evaluación de las necesidades de todo un grupo requiere de estudios de campo largos y costosos, que rara vez son posibles en el contexto de este tipo de visitas. Actualmente un subgrupo de nuestro grupo de trabajo está desarrollando un instrumento sencillo para medir algunos indicadores esenciales.

\section{Evaluación de Sistemas de Salud Mental}

La evaluación de los sistemas de salud mental debería, desde un punto de vista ideal, incluir la estructura (la descripción de los diferentes servicios), el proceso (el conocimiento técnico y el marco ético dentro del cual se ofrecen los servicios) y los resultados. Las "naciones clientes" pueden proporcionar algunos datos fiables pero sólo se puede obtener información más sofisticada a través de investigaciones ad hoc. Sin embargo, rara vez están garantizados el tiempo, el presupuesto y el suficiente compromiso de los clientes.

\section{Indicadores}

Se han descrito demasiados criterios, demasiadas normas, estándares e indicadores en el campo de la Salud Mental. La tendencia actual consiste en seleccionar unos pocos "indicadores" factibles, que son variables medibles y bien definidas en relación con los resultados de las actividades médicas. En la reunión de los centros europeos colaboradores de la OMS (Lille 2001) arriba mencionada, se presentó un amplio estudio que está siendo llevado a cabo por un equipo de investigadores de Londres con la finalidad de obtener indicadores simples y fiables (Thornicroft y Tansella 1999).
Además, un sub-grupo de nuestro grupo de trabajo de la OMS está trabajando sobre la selección de cinco indicadores generales de salud mental a añadir a la base de datos HFA-21 de la OMS. Es de esperar que estos esfuerzos nos conduzcan a disponer de instrumentos simples y ampliamente aceptados que podamos utilizar en el futuro.

\section{Evaluación de un Servicio Psiquiátrico}

El grupo de trabajo tiene eventualmente que concentrarse en la evaluación de un determinado servicio psiquiátrico. Tiene que incluir la descripción del entorno, el manual de procedimientos, la calidad de la acogida ofertada, la motivación de los profesionales, etc. Todos estos aspectos pueden ser fácilmente evaluados mediante los instrumentos existentes.

Para el grupo de trabajo, los indicadores clínicos cuantitativos (recursos, funcionamiento de actividad) son fácilmente accesibles en la mayoría de los países. Sin embargo, es más difícil obtener indicadores cualitativos sin estudios específicos. En realidad, la calidad clínica de los servicios psiquiátricos ha sido estudiada durante años por muchas organizaciones que han propuesto diversos manuales de acreditación y se han creado numerosas comisiones sobre la acreditación de centros psiquiátricos (WHO 1998, 1999). El realizar este tipo de estudios en nuestras evaluaciones (WHO 1999b), requiere energía, habilidades y presupuesto adecuados, rara vez disponible en las circunstancias actuales nuestras visitas nacionales.

\section{Detección de problemas clínicos}

Durante nuestras visitas, a través, por ejemplo, del estudio de las historias clíni- 
cas, hemos identificado diversos problemas en relación con la calidad clínica, que 1lamaron nuestra atención sobre la existencia de insuficiencias en algunos servicios o sistemas que requerirían ser investigadas con más profundidad. Sin embargo, la mayoría de los problemas sólo podrían ser estudiados adecuadamente mediante un proceso de evaluación in situ más largo, lo que no ha sido el caso en nuestras vivitas hasta ahora.

\section{Conclusión}

Durante los tres años de trabajo, el grupo de trabajo, siguiendo las recomendaciones HEALTH 21 (WHO 1998) de la Oficina Regional, que enfatizaban la necesidad de una cooperación intersectorial, ha podido acumular conocimientos habilidades sobre la "evaluación de la Salud Mental basados en las pruebas" (evidence based), escribir informes sobre el estado de la cuestión en Europa y desarrollar estrategias para llevar a cabo un número de evaluaciones en siete países de Europa del Este. Las visitas que aquí se han mencionado mostraron la existencia de importantes desigualdades entre estos países y otros más desarrollados, en cuanto a determinantes de salud socioeconómicos, estilos de vida, recursos en Salud Mental y acceso a los servicios.

El grupo de trabajo ha discutido muchos instrumentos para la evaluación de las necesidades de la población, de los recursos y de la eficacia de los sistemas y algunos fueron utilizados durante las evaluaciones pero muchos resultaron inadecuados. Se ha de hacer en el futuro un gran esfuerzo para ponerse de acuerdo sobre procedimientos simples y fiables de evaluación de estos parámetros y de control de la evolución.
Los límites de tiempo y de presupuesto condicionan en gran medida la tarea solicitada pero en muchos casos pudo hacerse una estimación global de los modelos de asistencia, del sistema de cuidados, de sus límites, de las necesidades de la población y de la provisión de cuidados actual. En algunos casos se hicieron algunas recomendaciones para un plan estratégico de cara a un sistema local de servicios de Salud Mental. En general, se ha observado tras las visitas un aumento del interés y una sensibilidad hacia las cuestiones de salud mental por parte de los políticos de los diferentes países. Algunos han solicitado una evaluación en profundidad de la Salud mental, que podría llevar a introducir modificaciones en los servicios a nivel local y eventualmente establecer un ciclo de control y de revisión.

Sin embargo, la mayoría de los políticos y de los administradores se muestran reticentes en cuanto a cuestiones de Salud Mental porque argumentan que no existe una relación clara entre diagnóstico y necesidades y que existen dificultades en la evaluación de los resultados. Para mejorar la situación necesitaríamos proporcionar información simple y relevante a la población general, a los políticos, a los grupos de riesgo, a los pacientes y a sus familias, a los profesionales de la asistencia primaria, a los educadores, a los profesionales de la salud mental y a los administradores. Pero por supuesto, estas son intervenciones costosas y a largo plazo.

En parte debido a las actividades de este grupo de trabajo, la cooperación de la OMS con la UE está en una fase de desarrollo bilateral en temas psiquiátricos. Por ambos lados se han enfatizado el deseo de actividades sinérgicas, la coordinación del trabajo y el intercambio de experiencias así como la asistencia técnica mutua. Como esfuerzos bilaterales conjunto se han realizado un con- 
greso conjunto, una sesión de brain storming sobre la promoción de la Salud Mental y una conferencia europea ese tema. Por otro lado, la Oficina Regional ha estrechado los vínculos con los cuarteles generales de la OMS, con otras redes de la Oficina Regional, con grupos de defensa de los enfermos, con la Federación Mundial de la Salud Mental (WFMH) y con la Asociación Mundial de Psiquiatría (WPA).

\section{Bibliografía}

FREEMAN, H., HENDERSON, J., RUTZ, W. WHO/EURO Database on Trends in Mental Health Care (1992) - A Review and Summing Up. Stockholm: First European Meeting of National Counterparts for the WHO/EURO Mental Health Program; 20-22, January 2000 .

HARDMANN, E., JOUGHIN, C. Focus on Clinical audit in Child and Adolescent Mental Health Services. London: Royal college of Psychiatrists, 1998.

MATTSON MARLIN, R. (ed). Manual of Psychiatric Quality Assurance: a Report of the American Psychiatric Association Committee on Quality Assurance. Washington, D.C.: APA, 1992

SLADE, S., THORNICROFT, G., LOFTUS, L., et al. Camberwell Assessment of Need. London: Royal College of Psychiatrists, 1999.
STAKES. European Commission. Minimum Data Set of European Mental Health Indicators: Proposed Set of Mental Health Indicators, Definitions, Description and Sources, 2001

THORNICROFT, G., TANSELLA, M. The Mental Health Matrix: a Manual to Improve Services. Cambridge University Press, 1999.

WHO. Regional Office for Europe. Mental Health Program Position Paper. HPSA 010401/BG1, January 1999 (unpublished document).

WHO. Regional Office for Europe. Health 21 - health for all in the 21st century. Copenhagen: WHO, 1998.

WHO. Balancing Mental Health Promotion and Mental Health Care: a Joint World Health Organization/European Commission Meeting. Brussels: 22-24, April 1999.

WHO. Division of Mental Health and Prevention of Substance Abuse, Programme on Mental Health. Guidelines for the Promotion of Human Rights of Persons with Mental Disorders. Geneva: WHO, 1996.

WHO. Regional Office for Europe. Health 21 - health for all in the 21st century. Copenhagen: WHO, 1998.

Dirección para correspondencia:

Profesor José Guimón

Departmento de Psiquiatría

Hôpitaux Universitaires de Genève

2 ch. du Petit-Bel-Air

CH-1225 Chêne-Bourg

E-mail: jose.guimon@hcuge.ch

SUIZA 